\title{
Resonance and stability of higher derivative theories of a derived type
}

\author{
Dmitry S. Kaparulin \\ Physics Faculty, Tomsk State University, Lenina ave. 36, Tomsk 634050, Russia \\ and Lebedev Institute of Physics, Leninsky ave. 53, Moscow 119991, Russia \\ Simon L. Lyakhovich $^{*}$ and Oleg D. Nosyrev ${ }^{\dagger}$ \\ Physics Faculty, Tomsk State University, Lenina ave. 36, Tomsk 634050, Russia
}

(Received 1 April 2020; accepted 11 May 2020; published 2 June 2020)

\begin{abstract}
We consider the class of higher derivative field equations whose wave operator is a square of another self-adjoint operator of lower order. At the free level, the models of this class are shown to admit a twoparameter series of integrals of motion. The series includes the canonical energy. Every conserved quantity is unbounded in this series. The interactions are included into the equations of motion such that a selected representative in conserved quantity series is preserved at the nonlinear level. The interactions are not necessarily Lagrangian, but they admit the Hamiltonian form of dynamics. The theory is stable if the integral of motion is bounded from below due to the interaction. The motions are finite in the vicinity of the conserved quantity minimum. The equations of motion for fluctuations have the derived form with no resonance. The general constructions are exemplified by the models of the Pais-Uhlenbeck (PU) oscillator with multiple frequency and Podolsky electrodynamics. The example is also considered of stable non-Abelian Yang-Mills theory with higher derivatives.
\end{abstract}

DOI: 10.1103/PhysRevD.101.125004

\section{INTRODUCTION}

In 1950, Pais and Uhlebeck first considered the class of relativistic higher derivative theories whose wave operator is a polynomial in the another formally self-adjoint operator of lower order [1]. Such models can be termed the theories of derived type. The general derived model is specified by the constant coefficient polynomial, termed the characteristic polynomial, and the lower order operator, being the primary operator. As far as the wave equations are determined modulo multiplication by a nonzero constant, only the structure of the characteristic polynomial roots is relevant. Depending on the structure of roots and primary operator, the setting of a derived model can describe many long-known higher derivative field theories; see [2] for review. The example is the Podolsky electrodynamics [3]; the conformal gravity theories in various dimensions [4,5] also fall in this class. The extended Chern-Simons [6] usually serves as prototype of a gauge derived model in the space-time dimension three. The Pais-Uhlenbeck (PU)

\footnotetext{
*sll@phys.tsu.ru

odnosyrev@phys.tsu.ru

${ }^{*}$ dsc@phys.tsu.ru
}

Published by the American Physical Society under the terms of the Creative Commons Attribution 4.0 International license. Further distribution of this work must maintain attribution to the author(s) and the published article's title, journal citation, and DOI. Funded by SCOAP ${ }^{3}$. oscillator [1] is the best known example of the derivedtype higher derivative mechanical model.

The derived theories are often considered in the context of studying of various aspects of higher derivative dynamics, including the problem of stability. There is a vast literature on the subject; see the reviews in [7-10] and references therein. The recent studies demonstrate that the higher derivative theories are not necessary unstable, even though the canonical energy is unbounded. ${ }^{1}$ The stability has been studied from several viewpoints. The non-Hermitian quantum mechanics is used to construct stable quantum theory of the PU model [11-13]. The same problem is solved by means of alternative Hamiltonian formulations in [14,15]. The stability of classical paths is studied by numerical simulations and analytical methods in [16-19]. In the paper [20], the class of metastable interaction vertices with logographically small runaway speed is proposed. The metastability phenomenon has been recently noticed in the $R^{2}$ gravity in the energy range below the certain threshold [21]. The structure of symmetries and conservation laws of derived type models is studied in [22,23]. It is shown that the bounded integrals of motion can exist in these theories that stabilizes the higher derivative dynamics. This makes the derived type higher derivative systems stable indeed, not just having an island of stability.

\footnotetext{
${ }^{1}$ Gauge theories with higher derivatives can have on shell bounded energy.
} 
The conclusions about the stability of higher derivative models are mostly related to the theories with simple roots of a characteristic polynomial. The models with multiple characteristic polynomial roots usually demonstrate instability already at the free level. The simplest example of this phenomenon is provided by the fourth order PU oscillator with a multiple frequency. The oscillations resonate, so the motion is unbounded. The theory with resonance has a bounded from below the integral of motion, though it does not lead to stability [24]. The alternative Hamiltonian formulations for PU model with resonance are canonically equivalent, and all the possible Hamiltonians are unbounded [14]. The multiple root in the characteristic polynomial has been previously considered as a indication of instability unless the corresponding degree of freedom is a pure gauge. As the example of this phenomenon we mention the extended Chern-Simons theory with a multiplicity two zero root which is stable due to the gauge invariance [23].

In papers [25-27], the problem of inclusion of stable interactions is studied from the viewpoint of consistent deformation of equations of motion and conserved quantities. It is demonstrated that the higher derivative theories admit the class of interactions such that preserve a selected conserved quantity of the free model. If a bounded from below quantity is conserved at the interacting level, the dynamics of the nonlinear theory is stable. The interacting equations are non-Lagrangian, but the dynamics is explicitly covariant, and it still admits the Hamiltonian formulation. For construction of interaction, it is critical that the free theory is stable. The interaction just preserves the model stability. In the class of derived theories with resonance at the free level, the free evolution is unbounded. This means that the interactions have to stabilize originally unstable theory. The phenomenon of stabilization of dynamics by the interaction is well-known in the theories without higher derivatives. For example, the motions of mechanical system can bounded in the vicinity of the unstable equilibrium position once the higher order corrections are accounted for the energy.

In the present paper, we construct the stable interactions for the class of higher derivative theories of derived type with a single multiplicity two root of the characteristic polynomial. The wave operator of the general model is given by the square of another formally self-adjoint operator of lower order. The simplest model is the PU oscillator with a resonance solution at the free level. We exploit the idea of stabilization of dynamics by means of interaction. We demonstrate that the interactions can be introduced such that the unbounded energy of the free model becomes bounded from below on the account of the interactions. The dynamics turns out stable in the vicinity of the energy minimum just by the reasons of energy conservation. The equations of motion for fluctuations have derived type without a resonance. The motions are stable in the range of energies below a certain level. Above this level, the dynamics becomes singular. All the proposed stable interactions are non-Lagrangian, but the equations still admit a Hamiltonian formulation. The theory of small fluctuations in the vicinity of stationary solution is Hamiltonian with a bounded Hamiltonian.

The general construction is illustrated by two examples: the PU oscillator with a multiple frequency, and Podolsky electrodynamics. For the PU oscillator, we detail the structure of the conserved quantities at the free and interacting level. We notice that the free theory admits a twoparameter series of conserved quantities. The canonical energy is included in the series. All the conserved quantities in the series are unbounded. The particular class of interactions that preserves a selected representative in the series of conserved quantities is proposed. If the interaction makes the integral of motion bounded, the nonlinear model turns out stable. The equations of motion for small fluctuations and the upper bound are identified for the energy such that the model admits the stable paths. The Podolsky electrodynamics provides a field-theoretical example of a similar phenomenon. In this higher derivative theory with unbounded energy of free model, we find the class of stable interactions with complex scalar field. The non-Abelian generalization of the proposed interactions are discussed.

The article is organized as follows. In the next section, we consider the conservation laws of the free PU model with multiple frequency. We construct a two parameter series of integrals of motion which includes the canonical energy. The general representative in the series can be interpreted as the energy of a certain model without higher derivatives. Proceeding from this interpretation, we construct the class of stable interactions that correspond to the (not necessary Lagrangian) theory with higher derivatives. In Sec. III, we observe that the theories with bounded energy are stable in certain sense. We identify all the equilibrium positions of theory. The equilibrium position at the origin is unstable, while the additional equilibrium positions can be stable. The motions are biharmonic oscillations in the vicinity of the stable equilibrium positions. The oscillation frequencies are different. The estimate for the upper limit of energy is given such that leads to bounded trajectories. In Sec. IV, we consider the issue of stability from the viewpoint of Hamiltonian formalism. It is shown that the interacting theory admits the Hamiltonian formulation with a bounded from below Hamiltonian. In Sec. V, we consider the field theoretical example-the Podolsky electrodynamics. In Sec. VI, the further generalizations of the proposed constructions are discussed. In the Conclusion, we summarize the results.

\section{THE PU OSCILLATOR WITH A MULTIPLE FREQUENCY}

Consider the mechanical model with two coordinates $x(t), y(t)$ and the action functional, 


$$
\begin{aligned}
S[x(t), y(t)] & =\int L d t, \\
L & =m\left(\ddot{x} \dot{y}-\omega^{2}\left(x y-\frac{1}{2} y^{2}\right)+\frac{1}{2} k\left(\dot{y}^{2}-\omega^{2} y^{2}\right)\right) .
\end{aligned}
$$

The action includes dimensionless parameter $k$, while $m$ is a mass, and $\omega$ is a frequency. The Lagrange equations read

$$
\begin{aligned}
& \frac{\delta S}{\delta x}=-m\left(\ddot{y}+\omega^{2} y\right)=0, \\
& \frac{\delta S}{\delta y}=-m\left(\ddot{x}+\omega^{2}(x-y)\right)-m k\left(\ddot{y}+\omega^{2} y\right)=0 .
\end{aligned}
$$

These equations can be equivalently written as

$$
\ddot{y}+\omega^{2} y=0, \quad \ddot{x}+\omega^{2} x=\omega^{2} y .
$$

Obviously, the parameter $k$ drops out of the equations, while it cannot be excluded from the action by adding any total derivative to the Lagrangian. In this sense, the Eq. (3) admit a one-parameter series of Lagrangians (1). This simple observation has consequences: given the series of actions, any symmetry of the equations leads to the series of the conserved quantities constructed by Noether theorem. This series is important for the inclusion of stable interactions.

The linear equations (3) obviously describe the resonating oscillations: $y$ harmonically oscillates with the frequency $\omega$, while the oscillator equation for $x$ includes the same frequency as in the solution for $y$. The latter is included as the "force" on the rhs of the equation for $x$. So the motion in the direction of $x$ is unbounded at free level. A different interpretation is possible for the same system (3): $y$ can be considered as an auxiliary variable because it can be expressed in terms of $x$ and $\ddot{x}$,

$$
y=x+\frac{\ddot{x}}{\omega^{2}}+\frac{1}{m \omega^{2}}\left(k \frac{\delta S}{\delta x}-\frac{\delta S}{\delta y}\right) \approx x+\frac{\ddot{x}}{\omega^{2}} .
$$

The symbol $\approx$ means the equality on the mass shell. Once the auxiliary variable $y$ is excluded by means of (4), the remaining coordinate $x$ obeys a single fourth order equation of the derived type,

$$
\dddot{x}+2 \omega^{2} \ddot{x}+\omega^{4} x=D^{2} x=0, \quad D=\left(\frac{d^{2}}{d t^{2}}+\omega^{2}\right) .
$$

It is the PU oscillator [1] with resonance. Equation (5) in itself comes from the action principle with higher derivatives,

$$
\begin{aligned}
S_{x}[x(t)] & =\int L_{x} d t, \\
L_{x}(x, \dot{x}, \ddot{x}) & =-\frac{1}{2 \omega^{2}}\left(\ddot{x}^{2}-2 \omega^{2} \dot{x}^{2}+\omega^{4} x^{2}\right) .
\end{aligned}
$$

As we see, the model (1) with any $k$ is equivalent to (5) at the level of equations of motion. The solutions, symmetries and conserved quantities are in one-to-one correspondence for the models (1) and (6). With this regard, one can consider (1) and (6) as two different representations of the same dynamics. The formula (4) expresses $y$ in terms of the derivatives of $x, \ddot{x}$, while $\dot{y}$ admits representation in terms of $\dot{x}, \dddot{x}$,

$$
\begin{array}{ll}
y=\omega^{-2} \ddot{x}+x, & \dot{y}=\omega^{-2} \dddot{x}+\dot{x} \Leftrightarrow \\
\ddot{x}=\omega^{2}(y-x), & \dddot{x}=\omega^{2}(\dot{y}-\dot{x}) .
\end{array}
$$

The substitution of $y$ in terms of $x$ and $\ddot{x}$ takes Eqs. (2)-(5), and vice versa.

We shall seek for the stable interactions such that the equivalence still exists between the higher derivative representation and the first order form of this dynamics at the level of equations of motion. We shall not require, however, the higher derivative equations to remain Lagrangian upon inclusion of interactions. The key role for our construction is played by the series of conserved quantities parametrized by $k$ involved (1). All these conservation laws are generated by the same symmetry, as we have already mentioned.

The action functional (1), being essentially $k$-dependent, is invariant with respect to the time translations. The canonical energy of the model is the conserved quantity,

$$
J=m\left(\dot{x} \dot{y}+\omega^{2}\left(x y-\frac{1}{2} y^{2}\right)+\frac{1}{2} k\left(\dot{y}^{2}+\omega^{2} y^{2}\right)\right) .
$$

This expression represents the energy in terms of the coordinates $x, y$. It explicitly depends on the parameter $k$. Given the connection between $y$ and $x$ (4), the conserved quantity can be represented in terms of a coordinate $x$ and its higher derivatives,

$$
\begin{aligned}
J= & m\left\{\omega^{-2} \dot{x} \dddot{x}-\frac{1}{2} \omega^{-2} \ddot{x}^{2}+\dot{x}^{2}+\frac{1}{2} \omega^{2} x^{2}\right. \\
& \left.+\frac{1}{2} k\left(\left(\omega^{-2} \dddot{x}+\dot{x}\right)^{2}+\omega^{2}\left(\omega^{-2} \ddot{x}+x\right)^{2}\right)\right\} .
\end{aligned}
$$

As is seen, the constant $k$ is involved in the integral of motion, while the equation of motion (5) is independent of $k$. This means that $k$-dependent and $k$-independent terms conserve separately, i.e., 


$$
\begin{aligned}
& \frac{d}{d t}\left(\omega^{-2} \dot{x} \dddot{x}-\frac{1}{2} \omega^{-2} \ddot{x}^{2}+\dot{x}^{2}+\frac{1}{2} \omega^{2} x^{2}\right) \approx 0, \\
& \frac{d}{d t}\left(\left(\omega^{-2} \dddot{x}+\dot{x}\right)^{2}+\omega^{2}\left(\omega^{-2} \ddot{x}+x\right)^{2}\right) \approx 0 .
\end{aligned}
$$

Equations (8), (9) determine a two-parameter series of the conserved quantities of the PU theory with resonance. Each representative in the series can be considered as the canonical energy of a certain representative of the action functional series (1). This interpretation is not generally true at the higher derivative formalism (5). The canonical energy of the higher derivative theory (6) corresponds to the first entry in the series (9).

The integrals of motion (10) do not result in the stability of the dynamics. The series (8) involves the dynamical variable $x$ in a linear way. Once $x, y$ and $\dot{x}, \dot{y}$ admit independent initial data, the corresponding conserved quantity is obviously unbounded. As the classical energy does not have a minimum, one can expect the unbounded spectrum of Hamiltonian at quantum level, and the "Ostrogradski ghosts" emerge. Another reason is that the conservation law (8) does not restrict the motion to the vicinity of the critical point $x=y=0$. The general solution of the equations (2) read

$$
\begin{aligned}
& x(t)=\omega A t \sin (\omega t+a)+B \sin (\omega t+b), \\
& y(t)=2 A \cos (\omega t+a),
\end{aligned}
$$

where $A, B$ and $a, b$ are the arbitrary integration constants. The amplitude of the first oscillation (11) is linearly growing with time. The motion is finite only for the special solutions with $A=0$. The substitution of the general solution (11) into (8) gives the expression for the conserved quantity in terms of initial data,

$$
\left.J\right|_{x=x(t), y=y(t)}=2 \omega^{2}\left(A B \sin (b-a)+(k-1) A^{2}\right) .
$$

For any fixed value of the parameter $k$, the condition $J=$ const does not restrict the amplitudes of oscillations. The motion is unbounded for any value of $J$. The latter fact explains the instability of model (5) from the viewpoint of structure of its classical paths. Obviously, the absence of lower bound of the general representative of the energy series (8) is a source of the instability. However, the stabilization of dynamics is possible upon the inclusion of interaction if the conserved quantity is bounded from below in a vicinity of the critical point.

At the end of this section, we would like to make a remark on the role of the parameter $k$ in the series of action functionals (1) and integrals of motion (8). Obviously, the different representatives in these series are connected by the coordinate transformation,

$$
x \mapsto x^{\prime}=x+\frac{1}{2} s y, \quad y \mapsto y^{\prime}=y,
$$

where $s$ is the real constant parameter. The transformation with $s=-k$ brings $k$ to zero in a general representative in (1), (8). In terms of the higher derivative description, this transformation does not correspond to any local change of the coordinate $x$. Instead, we have a nonlocal transformation,

$$
x \mapsto x^{\prime}=x+\frac{1}{2} s\left(\omega^{-2} \ddot{x}+x\right),
$$

which is not invertible in the class of local coordinate changes of the coordinate $x$. In particular, the variational principle (1) with $k \neq 0$ does not correspond to any variational principle with higher derivatives and single dynamical variable $x$. This fact has several consequences at the nonlinear level and in the Hamiltonian formalism, which are discussed in the next two sections.

\section{STABLE INTERACTIONS}

In this section, we consider the inclusion of interaction in the Lagrangian (1) such that the coordinate $y$ can be still excluded on shell in terms of $x, \dot{x}, \ddot{x}$, though not necessarily in the same way as it has been done in the free theory (4). This would mean that the interactions in the first order theory (1) correspond to the local interactions in the higher derivative equation (5), though the interaction vertex is not necessarily Lagrangian in the higher derivative setup.

The simplest option is to consider the class of interactions in the model (1) where the coordinate $y$ is expressed from the same combination of the Lagrangian equations as in the free theory (4). In the slightly different wording, this means that at the interacting level, the same linear combination of Lagrangian equations defines $y$ as function of $x, \dot{x}, \ddot{x}$,

$$
y-f(x, \dot{x}, \ddot{x})=\frac{1}{m \omega^{2}}\left(\frac{\delta S}{\delta y}-k \frac{\delta S}{\delta x}\right),
$$

where $f(x, \dot{x}, \ddot{x})$ is a function of the coordinate $x$ and its derivatives. This function can be nonlinear, unlike the free model (4). The most general Lagrangian that has the property (15) reads

$$
\begin{aligned}
L= & m\left(\dot{x} \dot{y}-\omega^{2}\left(x y-\frac{1}{2} y^{2}\right)+\frac{1}{2} k\left(\dot{y}^{2}-\omega^{2} y^{2}\right)+\right. \\
& +U(x, \dot{x})+V(z, \dot{z})),
\end{aligned}
$$

where $U(x, \dot{x}), V(z, \dot{z})$ are the functions of the variables $x$, $z \equiv x+k y$ and its first time derivatives $\dot{x}, \dot{z}$. The interaction potentials $U, V$ are assumed at least cubic in the variables. It terms of the potentials, the coordinate $y$ is defined on shell as follows:

$$
y=f(x, \dot{x}, \ddot{x}) \equiv \frac{1}{\omega^{2}}\left(\ddot{x}+\omega^{2} x+k\left(\frac{\partial U}{\partial x}-\frac{d}{d t} \frac{\partial U}{\partial \dot{x}}\right)\right) .
$$


The function $V(x, \dot{x})$ does not contribute to this equation. The higher derivative equation of motion for a single coordinate $x$ has the following form:

$$
\begin{gathered}
\frac{1}{\omega^{2}}\left(\frac{d}{d t^{2}}+\omega^{2}\right)^{2} x+\left((k-1)+\frac{k}{\omega^{2}} \frac{d^{2}}{d t^{2}}\right)\left(\frac{\partial U}{\partial x}-\frac{d}{d t} \frac{\partial U}{\partial \dot{x}}\right) \\
-\left.\left(\frac{\partial V}{\partial z}-\frac{d}{d t} \frac{\partial V}{\partial \dot{z}}\right)\right|_{z=x+k f(x, \dot{x}, \dot{x})}=0
\end{gathered}
$$

where the function $f(x, \dot{x}, \ddot{x})$ denotes the right-hand side of Eq. (17). The obtained equation is the obvious deformation of the free PU theory equation (5). The class of interacting theories (5) is not invariant with respect to the transformation (13). This automatically means that the different representatives of the free action functional series (1) give rise to different classes of interacting theories, which are unconnected to each other by any change of coordinates.

Only one representative of the integral of motion series (8) conserves at the interacting level. It is given by the canonical energy of the action functional (16),

$$
\begin{aligned}
J= & m\left\{\dot{x} \dot{y}+\omega^{2}\left(x y-\frac{1}{2} y^{2}\right)+\frac{1}{2} k\left(\dot{y}^{2}+\omega^{2} y^{2}\right)\right. \\
& \left.+\dot{x} \frac{\partial U}{\partial \dot{x}}-U+\dot{z} \frac{\partial V}{\partial \dot{z}}-V\right\} .
\end{aligned}
$$

This quantity admits representation in two equivalent forms. First, the quadratic form of this expression can be brought to the diagonal form. In so doing, we assume ${ }^{2}$ that $k(k-1) \neq 0$,

$$
\begin{aligned}
J= & m\left\{\frac{k}{2}\left(\dot{y}+\frac{1}{k} \dot{x}\right)^{2}+\frac{(k-1) \omega^{2}}{2}\left(y+\frac{1}{k-1} x\right)^{2}\right. \\
& \left.-\frac{1}{2 k} \dot{x}^{2}-\frac{\omega^{2}}{2(k-1)} x^{2}+\dot{x} \frac{\partial U}{\partial \dot{x}}-U+z \frac{\partial V}{\partial \dot{z}}-V\right\} .
\end{aligned}
$$

Second, the coordinate $y$ can be expressed in terms of $x$ and its derivatives by means of Eq. (17). In this way, we arrive to the conservation law of the higher derivative theory (18),

$$
\begin{aligned}
J= & m\left\{\frac{k}{2}\left(\frac{k \omega^{-2} \dddot{x}+(k+1) \dot{x}}{k}+\frac{k}{\omega^{2}} \frac{d}{d t}\left(\frac{\partial U}{\partial x}-\frac{d}{d t} \frac{\partial U}{\partial \dot{x}}\right)\right)^{2}\right. \\
& +\frac{(k-1) \omega^{2}}{2}\left(\frac{(k-1) \omega^{-2} \ddot{x}+k x}{k-1}+\frac{k}{\omega^{2}}\left(\frac{\partial U}{\partial x}\right.\right. \\
& \left.\left.-\frac{d}{d t} \frac{\partial U}{\partial \dot{x}}\right)\right)^{2}-\frac{1}{2 k} \dot{x}^{2}-\frac{\omega^{2}}{2(k-1)} x^{2}+\dot{x} \frac{\partial U}{\partial \dot{x}}-U \\
& \left.+\left.\left(z \frac{\partial V}{\partial \dot{z}}-V\right)\right|_{z=x+k f(x, \dot{x}, \ddot{x})}\right\} .
\end{aligned}
$$

\footnotetext{
${ }^{2}$ The assumption does not restrict generality. As we demonstrate below, only interactions with $k>1$ can be stable.
}

As is seen from Eqs. (20) and (21), the unbounded contributions come from the terms with squares of $x$ and $\dot{x}$. The integral of motion of the interacting theory is bounded if the contributions from the interaction potentials $U, V$ compensate the unbounded terms. The simplest example of interactions that meet the stability condition reads

$U(x, \dot{x})=\frac{1}{4 \omega^{2}} \alpha \dot{x}^{4}+\frac{1}{2} \beta x^{2} \dot{x}^{2}-\frac{1}{4} \gamma \omega^{2} x^{4}$,

$V(x, \dot{x}, \ddot{x})=0$,

where the constants $\alpha, \beta, \gamma$ represent the interaction parameters.

In this article, we do not explore the dynamics of nonlinear model (18) with the most general potentials $U, V$ such that lead to stability. For the sake of technical simplicity, we mostly focus on the particular class of interaction potentials (22). As we observe below, this class is wide enough to illustrate the general properties of the dynamics at the nonlinear level. Given the specific potentials, equation of motion (18) reads

$$
\begin{aligned}
(1- & \left.k\left(3 \alpha \omega^{-2} \dot{x}^{2}+\beta x^{2}\right)\right) \dddot{x}-6 k\left(3 \alpha \omega^{-2} \dot{x} \ddot{x}+\beta x \dot{x}\right) \dddot{x} \\
& +\left(2 \omega^{2}+3 \alpha\left((1-k) \dot{x}^{2}-6 k \omega^{-2} \ddot{x}^{2}\right)+\beta\left((1-k) \omega^{2} x^{2}\right.\right. \\
& \left.\left.-k\left(4 x \ddot{x}+7 \dot{x}^{2}\right)\right)-3 \gamma k \omega^{2} x^{2}\right) \ddot{x}+(\beta(1-k)-6 \gamma k) \\
& \times \omega^{2} x \dot{x}^{2}+\omega^{4}\left(x-\gamma(k-1) x^{3}\right)=0 .
\end{aligned}
$$

The integral of motion of the nonlinear theory (23) is given by the expression (21) with the interaction potential (22). The explicit computation gives

$$
\begin{aligned}
J= & m\left\{\frac { k } { 2 } \left(\frac{k \omega^{-2} \dddot{x}+(k+1) \dot{x}}{k}-\frac{k}{\omega^{2}} \frac{d}{d t}\left(3 \alpha \omega^{-2} \dot{x}^{2} \ddot{x}\right.\right.\right. \\
& \left.\left.+\beta\left(x^{2} \ddot{x}+x \dot{x}^{2}\right)+\gamma \omega^{2} x^{3}\right)\right)^{2} \\
& +\frac{(k-1) \omega^{2}}{2}\left(\frac{(k-1) \omega^{-2} \ddot{x}+k x}{k-1}-\frac{k}{\omega^{2}}\left(3 \alpha \omega^{-2} \dot{x}^{2} \ddot{x}\right.\right. \\
& \left.\left.+\beta\left(x^{2} \ddot{x}+x \dot{x}^{2}\right)+\gamma \omega^{2} x^{3}\right)\right)^{2}-\frac{1}{2(k-1)} \omega^{2} x^{2} \\
& \left.-\frac{1}{2 k} \dot{x}^{2}+\frac{3}{4 \omega^{2}} \alpha \dot{x}^{4}+\frac{1}{2} \beta x^{2} \dot{x}^{2}+\frac{1}{4} \gamma \omega^{2} x^{4}\right\} .
\end{aligned}
$$

From the viewpoint of stability, the presence or absence of lower bound of energy is relevant. The first two lines of equation (24) involve total squares. These terms give positive contributions to energy if $k>1$ irrespectively to the specifics of interaction. The third line includes the interaction energy. At the free level it is unbounded. Once 
the interaction is included, it becomes bounded if the coupling constants $\alpha, \beta, \gamma$ are positive.

Depending on the structure of the interaction term in the integral of motion, the nonlinear theory can be stable or unstable. If the level surfaces of constant energy are bounded in the phase-space, the motions are finite for all the initial data. It is the case of the globally stable theory. The globally stable interactions are admitted by the PU oscillator with a nondegenerate frequency spectrum [25,28]. For examples of stable interactions in the field theory we refer to articles $[2,26,27]$. There is no analogous way to include the globally stable interaction for the PU oscillator with a multiple frequency. Under the less restrictive assumptions, the energy can be an unbounded function which admits a local minimum. Then, the motions are stable in the vicinity of the energy minimum. This is a case of so-called stability island. The models with the stability island are stable in the range of energies below a certain limit. The highest value of energy with a bounded isoenergetic surface determines the upper energy limit for the stable paths. In principle, the existence of a stability island is sufficient for the construction of quantum theory with metastable states and a well-defined vacuum state. The precedents are known of this type for the higher derivative systems $[8,18]$, though not with a resonance at free level. We see that the concept of an island of stability also suits well for the dynamics of interacting theory (23). The free model (5) is unstable. To get the stability in the interacting theory, the model should have a (local) minimum of energy due to interaction. The motions are bounded in the vicinity of the stable equilibrium position just by virtue of the energy conservation law.

Depending on the value of the interaction parameters $\alpha$, $\beta, \gamma$, the theory (23) may have one or three stationary solutions. The case $\gamma(k-1) \geq 0$ is not interesting because the model (23) has a single unstable equilibrium position at the origin. If $\gamma(k-1)>0$, we have two nonzero stationary solutions,

$$
x= \pm \frac{1}{\sqrt{\gamma(k-1)}} .
$$

They can be stable or unstable depending on the values of the interaction parameters. Introduce the special notation for the fluctuation in the vicinity of the equilibrium position,

$$
u=x \mp \frac{1}{\sqrt{\gamma(k-1)}} .
$$

The decomposition of the integral of motion (24) in the vicinity of nonzero stationary solution reads

$$
\begin{aligned}
J= & m\left\{-\frac{\omega^{2}}{4 \gamma(k-1)^{2}}\right. \\
& +\frac{1}{2 k}\left(\frac{1}{\omega^{2}}\left(1-\frac{\beta k}{\gamma(k-1)}\right) \dddot{u}-\frac{2 k^{2}+1}{k-1} \dot{u}\right)^{2} \\
& +\frac{\omega^{2}}{2(k-1)}\left(\frac{1}{\omega^{2}}\left(1-\frac{\beta k}{\gamma(k-1)}\right) \ddot{u}-\frac{2 k}{k-1} u\right)^{2} \\
& \left.+\frac{1}{2 k}\left(\frac{\beta k}{\gamma(k-1)}-1\right) \dot{u}^{2}+\frac{\omega^{2}}{k-1} u^{2}+\ldots\right\} .
\end{aligned}
$$

The dots denote cubic and higher terms in $u$ and its derivatives. The first term defines the value of energy at the stationary solution. We introduce the special notation for this value,

$$
J_{\min }=-\frac{m \omega^{2}}{4 \gamma(k-1)^{2}} .
$$

This value is negative for $\gamma>0, k>1$. The stability properties of the equilibrium position are determined by signature of the quadratic form in the decomposition (27). The energy has a minimum if all the coefficients at squares are positive,

$$
k-1>0, \quad \gamma>0, \quad \beta k-\gamma(k-1)>0 .
$$

Hereinafter, it is assumed that the stability conditions are satisfied. In particular, we suppose below that $\beta, \gamma>0$. The parameter $\alpha$ is not involved in the stability conditions because the $\dot{x}^{4}$ term cannot influence the motions in the vicinity of the equilibrium position. However, this term influences the stability properties of the model at higher energies.

The model (23) cannot be globally stable. The stable interactions imply the positive values of the interaction parameters $\beta, \gamma$. Once this parameters are positive, the coefficient at the highest derivative term in the equation of motion can vanish,

$$
1-k\left(3 \alpha \omega^{-2} \dot{x}^{2}+\beta x^{2}\right)=0
$$

On this phase-space surface, the conditions of existence and uniqueness of solution to the Cauchy problem for equation (23) are violated. Our analysis shows that the relations (23) and (30) are inconsistent. This means that neither true trajectory can be transverse to this surface, nor the classical path can lie on the phase-space submanifold (30). The only alternative is that true trajectories begin or end in the vicinity of the surface (30). In the globally stable theories, the classical path are complete. ${ }^{3}$ It is not possible for the system (23) as we see. The complete trajectories can

\footnotetext{
${ }^{3}$ The path $x(t)$ is complete if it is defined for the real value of time $-\infty<t<+\infty$.
} 
exist if the certain level energy surface defined by (24) has no intersection with the phase-space submanifold (30). The stationary solutions (25) are examples of complete paths. They do not lie on the surface (30) because conditions (25) and (30) are contradictory. The classical paths in the vicinity of stable equilibrium position have a good chance to be complete. The level surfaces of integral of motion (24) are compact and lie in the neighborhood of the equilibrium position with no intersection with the phase-space submanifold (30) if the energy value is sufficiently small. The biggest compact isoenergetic surface (24) that has no intersection with the phase-space submanifold (30) determines the border of stability island. The brief summary of the above is the following. The stable paths of the system (23) are localized in two islands of stability, which lie in the vicinity of nonzero stationary solutions (25). The existence of singular surface (30) is the obstruction to the global stability for the class of interactions (23).

Consider the dynamics of small fluctuations in the vicinity of the nonzero stationary solution (25). The linearization of equation (18) in the vicinity of solution (25) reads

$$
\left(\frac{\beta k}{\gamma(k-1)}-1\right) \dddot{u}+\left(\frac{\beta}{\gamma}+\frac{k+2}{k-1}\right) \omega^{2} \ddot{u}+2 \omega^{4} u=0,
$$

where $u$ is the fluctuation. By construction, the equation of motion has the PU form. The frequencies of oscillations are determined by the interaction parameters and the constant $k$. The stability conditions (29) imply that all the coefficients of equation are positive, so the roots of characteristic equations are complex. The frequencies of oscillations for the model (31) read

$$
\begin{aligned}
\omega_{ \pm} & =\omega \sqrt{\frac{\beta(k-1)+\gamma(k+2) \pm \sqrt{D}}{2(k \beta-\gamma(k-1))}}, \\
D & =(\beta(k-1)+\gamma(k+2))^{2}-8 \gamma(k-1)(\beta k-(k-1) \gamma) .
\end{aligned}
$$

The conditions (29) imply that the frequencies are not equal, so the system (31) has no resonance at the equilibrium position. The simplest way to see the fact follows. The Eq. (18) admit alternative formulation without higher derivatives (16). The resonance has the place if both the oscillators are one and the same frequency. This option is not possible because the system of two free oscillators with one and the same frequency does not allow reformulation in terms of higher derivative PU theory with a single dynamical variable. The solution to the equation of motion is a biharmonic oscillation,

$$
u=A \sin \left(\omega_{+} t+a\right)+B \sin \left(\omega_{-} t+b\right),
$$

where $A, B, a, b$ are integration constants. The biharmonic oscillation is a finite path, so the dynamics should be considered as stable. The account of interaction does not change conclusion of about the stability of motion. The argument is that the dynamics is localized on zero energy surface, which is compact for the energies slightly above the lower bound. All this means that the dynamics of nonlinear theory is stable in the vicinity of equilibrium position.

Let us now specify the stability island. The classical path is singular if the surfaces (24) and (30) are intersect. For regular path conditions (24) and (30) are inconsistent. The regularity condition is met in the range of energies,

$$
J_{\min } \leq J<J_{\max }
$$

where $J_{\min }$ is the minimal value of energy (28), and $J_{\max }$ is the minimal value of energy on the singular surface (30). Expressing the coordinate $x$ on the surface (30), we represent the energy (24) as the function of three variables,

$$
\begin{aligned}
J\left[x \mapsto \pm \sqrt{\left.\frac{1}{\beta}\left(\frac{1}{k}-\frac{3 \alpha}{\omega^{2}} \dot{x}^{2}\right)\right]}=\right. & m\left\{\frac{k}{2}\left[\left(\frac{k+1}{k}-\frac{3 \gamma}{\beta}\right) \dot{x}-\frac{6 \alpha k \dot{x} \ddot{x}^{2}}{\omega^{4}} \mp \frac{4 \dot{x} \ddot{x}}{\omega^{2}} \sqrt{\beta\left(\frac{1}{k}-\frac{3 \alpha}{\omega^{2}} \dot{x}^{2}\right)}+\frac{k}{\omega^{2}}\left(\frac{9 \gamma \alpha}{\beta}-\beta\right) \dot{x}^{3}\right]^{2}\right. \\
& +\frac{k^{3} \omega^{2}}{2 \beta(k-1)}\left(\frac{1}{k}-\frac{3 \alpha}{\omega^{2}} \dot{x}^{2}\right)\left[\frac{1}{k-1}-\sqrt{\beta\left(\frac{1}{k}-\frac{3 \alpha}{\omega^{2}} \dot{x}^{2}\right)}-\frac{\gamma}{\beta}\left(\frac{1}{k}-\frac{3 \alpha}{\omega^{2}} \dot{x}^{2}\right)\right]^{2} \\
& \left.+\frac{3 \alpha}{4 \omega^{2}}\left(\frac{3 \gamma \alpha}{\beta^{2}}-1\right) \dot{x}^{4}+\frac{3 \alpha}{2 \beta(k-1)}\left(1-\frac{\gamma(k-1)}{\beta k}\right) \dot{x}^{2}-\frac{\gamma \omega^{2}}{4 k^{2} \beta^{2}}\left(\frac{3 \beta k}{\gamma(k-1)}-1\right)\right\} .
\end{aligned}
$$

The domain of a function is $|\dot{x}|<\sqrt{1 / 3 k \alpha}$ for $\alpha>0$, and all the phase space for $\alpha \leq 0$. Otherwise the condition (30) is inconsistent. The arguments in squares in two first lines of (34) are independent initial data, which account the dependence of the energy on $\ddot{x}, \dddot{x}$. The value of these terms can be set zero irrespectively to the $x, \dot{x}$ terms of third line. The actual minimum of the function is given by the minimum of the quadratic form in $\dot{x}^{2}$ in the third line of the expression (34). Three different cases are summarized in the equation below, 


$$
J_{\max }=\left\{\begin{array}{l}
-\frac{m \gamma \omega^{2}}{4 \beta^{2} k^{2}}\left(\frac{2 \beta k}{\gamma(k-1)}-1\right), \\
\alpha=0 \quad \text { or } \quad \alpha \geq \frac{\beta^{2}}{3 \gamma} \quad \text { or } \quad 0<\alpha<\frac{\beta^{2}}{3 \gamma}, \quad \frac{\beta(\beta k-\gamma(k-1))}{k(k-1)\left(\beta^{2}-3 \gamma \alpha\right)} \geq \frac{1}{3 \alpha k} \\
-m \omega^{2}\left\{\frac{\gamma}{4 \beta^{2} k^{2}}\left(\frac{2 \beta k}{\gamma(k-1)}-1\right)-\frac{1}{2 \beta(k-1)}\left(1-\frac{\gamma(k-1)}{\beta k}\right)-\frac{1}{4}\left(\frac{3 \gamma \alpha}{\beta^{2}}-1\right)\right\} \\
0<\alpha<\frac{\beta^{2}}{3 \gamma}, \quad \frac{\beta(\beta k-\gamma(k-1))}{k(k-1)\left(\beta^{2}-3 \gamma \alpha\right)}<\frac{1}{3 \alpha k} \\
-\frac{m \gamma \omega^{2}}{4 \beta^{2} k^{2}}\left(\frac{2 \beta k}{\gamma(k-1)}-1\right)-\frac{3 m|\alpha| \omega^{2}}{4 k(k-1)^{2}} \frac{k \beta-\gamma(k-1)}{3 \gamma|\alpha|+\beta^{2}} \\
\alpha<0
\end{array}\right.
$$

The estimates in the right-hand side of this equation give the upper bound for the energy of the regular path. The surface $J=J_{\max }$ is the border of the stability island.

Let us summarize the results of the section. If the parameters of the model meet the condition (29), the nonlinear theory (23) has three equilibrium positions. The equilibrium position at the origin, being the critical point of the free theory, is unstable. The other two equilibrium points are stable. The motions are regular for energies in the range (33), where $J_{\min }, J_{\max }$ are given by relations (28), (35). The maximal energy of the regular trajectories defines the boundary of the stability island. The value $\alpha=0$ corresponds to the maximal possible size of the stability island [the first option in Eq. (35)]. The size of the stability island decreases for negative $\alpha$, which contributes a negative correction to the kinetic term, and also for small positive $\alpha$. The latter decrease of the energy limit may seem counterintuitive. We explain it by almost degeneracy of the energy quadratic form (27) at the equilibrium position (the coefficient at $\dot{u}$ is small). In this case, every small change of the model parameters can have a negative impact on stability.

\section{HAMILTONIAN FORMALISM}

Let us begin with the free PU theory. At first, consider the canonical Hamiltonian formalism for the theory with the action functional (1). The action involves the parameter $k$, which is not involved in the equivalent higher derivative theory (6), so we aim at clarifying the consequences of this ambiguity in Hamilitonian formalism.

Introduce the canonical momenta $p_{x}, p_{y}$ obeying the canonical,

$$
\begin{aligned}
p_{x}=m \dot{y}, \quad p_{y}=m(\dot{x}+k \dot{y}), \\
\{x, y\}=\left\{p_{x}, p_{y}\right\}=\left\{x, p_{y}\right\}=\left\{y, p_{x}\right\}=0, \\
\left\{x, p_{x}\right\}=\left\{y, p_{y}\right\}=1 .
\end{aligned}
$$

The Hamiltonian equations read

$$
\dot{z}^{i}=\left\{z^{i}, H\right\}, \quad z^{i}=\left(x, y, p_{x}, p_{y}\right)
$$

$H=\frac{1}{m}\left(p_{x} p_{y}+\frac{1}{2} k p_{x}^{2}\right)+m \omega^{2}\left(x y+\frac{k-1}{2} y^{2}\right)$.

The equivalence between the Hamiltonian equations (38), (39) and higher derivative PU theory (5) is easy to see: the momenta $p_{x}, p_{y}$ and auxiliary variable $y$ can be expressed in terms of the coordinate $x$ and its derivatives. The remaining dynamical variable $x$ obeys the higher derivative PU equation (5).

The Hamiltonians (39) depend on the parameter $k$. This dependence is due to the canonical transformation,

$$
\begin{aligned}
& x \mapsto x^{\prime}=x+\frac{1}{2} s y, \quad p_{x} \mapsto\left(p_{x}\right)^{\prime}=p_{x}-\frac{1}{2} s p_{y}, \\
& y \mapsto y^{\prime}=y, \quad p_{y} \mapsto\left(p_{y}\right)^{\prime}=p_{y},
\end{aligned}
$$

where $s$ is the transformation parameter, being a real number. The Ostrogradski canonical formulation [29] corresponds the choice $k=0$. The transformation (39) with $s=k$ connects the general Hamiltonian (39) with the canonical Hamiltonian by Ostrogradski. The subtlety is that the Hamiltonian formulations with $k \neq 0$ cannot be derived by means of the Legendre transformation of any higher derivative action functional. The paper [30] tells us that all the Hamiltonian formulations, being obtained by different ways of reduction of order in the Lagrangian, are connected by the canonical transformation, which does not involve the original coordinates. This is not the case at hands. The transformation (13) of single dynamical $x$ is not a change of the coordinate (14). It is some kind of hidden symmetry, being connected to the resonance. At the interacting level, the transformation (13) no longer preserves the equations of motion (23). In this case, the Legendre transformation of the action (16) allows us to construct the Hamiltonian formulations, which do not follow from the Ostrogradksi procedure for the higher derivative action (6). This Hamiltonian formulations can have bounded Hamiltonian even if the higher derivatives are involved at the free and interacting levels. That is why the Hamiltonian 
formulations (38), (39) are useful from the viewpoint of inclusion of interactions.

The Hamiltonian formulations (38), (39) can be rewritten in terms of the phase-space variables $x, \dot{x}, \ddot{x}, \dddot{x}$, being derivatives of $x$. The transformation law reads

$$
\begin{aligned}
& \dot{x}=\frac{1}{m}\left(p_{y}-k p_{x}\right), \quad \ddot{x}=\omega^{2}(x-y), \\
& \dddot{x}=\frac{1}{m} \omega^{2}\left((k+1) p_{x}-p_{y}\right) .
\end{aligned}
$$

The Hamiltonian (39) is given by the general representative of the conserved series (9),

$$
\begin{aligned}
H= & m\left\{\omega^{-2} \dot{x} \dddot{x}-\frac{1}{2} \omega^{-2} \ddot{x}^{2}+\dot{x}^{2}+\frac{1}{2} \omega^{2} x^{2}\right. \\
& \left.+\frac{1}{2} k\left(\left(\omega^{-2} \dddot{x}+\dot{x}\right)^{2}+\omega^{2}\left(\omega^{-2} \ddot{x}+x\right)^{2}\right)\right\} .
\end{aligned}
$$

The Poisson brackets of the phase-space variables $x, \dot{x}, \ddot{x}, \dddot{x}$ read

$$
\begin{aligned}
& \{x, \dot{x}\}=-\frac{1}{m} k, \quad\{\dot{x}, \ddot{x}\}=\{\dddot{x}, x\}=\frac{1}{m} \omega^{2}(k+1), \\
& \{\ddot{x}, \dddot{x}\}=\frac{1}{m} \omega^{4}(k+2), \quad\{x, \ddot{x}\}=\{\dot{x}, \dddot{x}\}=0 .
\end{aligned}
$$

The Hamiltonian formulation (42), (43) has been first proposed in [14] for the PU oscillator. The canonical equivalence of different Hamiltonian formulations has been also noticed in this article. The special feature of the Poisson bracket (43) is that the coordinate $x$ and its velocity $\dot{x}$ are conjugated if $k \neq 0$. In the Ostrogradski construction of the Hamiltonian formalism, the coordinate always Poisson commute to the velocity. This observation means the Hamiltonian formulation with $k \neq 0$ cannot be derived by the Legendre transformation of a higher derivative Lagrangian with a single dynamical variable $x$.

Let us now focus on the Hamiltonian formulation for the higher derivative equation with an interaction (22). Even though the interaction vertices are non-Lagrangian, the equivalent lower formulation admits the action principle (16). So, one can proceed from the action (16). Introduce the canonical momenta,

$$
p_{x}=m\left(\dot{y}+\alpha \omega^{-2} \dot{x}^{3}+\beta x^{2} \dot{x}\right), \quad p_{y}=m(\dot{x}+k \dot{y}) .
$$

From these equations the velocity $\dot{y}$ can be expressed in terms of $p_{x}, p_{y}$ and $\dot{x}$, while $\dot{x}$ is determined as a solution of algebraic equation,

$$
\begin{aligned}
& \dot{y}=\frac{1}{m} p_{x}-\alpha \omega^{-2} \dot{x}^{3}-\beta x^{2} \dot{x}, \\
& k \alpha \dot{x}^{3}+\left(k \beta x^{2}-1\right) \dot{x}+\frac{1}{m}\left(p_{y}-k p_{x}\right)=0 .
\end{aligned}
$$

This equation has a third order if $\alpha \neq 0$; otherwise it is linear. The cubic equation can be solved with respect to $\dot{x}$ by several methods, for example, the Cardano formula. Choosing the appropriate branch of the solution of Eq. (45), and computing the Hamiltonian, one can construct the Hamiltonian formulation for the interacting model (23). It should be outlined that for stable interactions the relative signs of the cubic and linear in $\dot{x}$ terms can be opposite. In this case, none of the branches of the solutions of the cubic equation are globally defined. This obstruction prevents the construction of globally defined Hamiltonian formalism in the theory (23). The number of solution branches in changed on the singular surface (30). If $\alpha=0$, the velocity is explicitly expressed from the Eq. (45). The expression for the Hamiltonian is given below,

$$
\begin{aligned}
H= & \frac{\beta x^{2}}{2 m}\left(\frac{p_{y}-k p_{x}}{\beta k x^{2}-1}\right)^{2} \\
& +\frac{1}{2 m} \frac{\left(k p_{x}+\left(\beta k x^{2}-2\right) p_{y}\right)\left(\beta x^{2} p_{y}-p_{x}\right)}{\left(\beta k x^{2}-1\right)^{2}} \\
& +m \omega^{2}\left(x y-\frac{k-1}{2} y^{2}+\frac{1}{4} \gamma x^{4}\right) .
\end{aligned}
$$

The Hamiltonian is singular at the surface (30). So, the Hamiltonian dynamics is not smooth in the vicinity of the singular surface even in the case $\alpha=0$.

Let us consider the Hamiltonian description of the dynamics of small fluctuations in the vicinity of the stable equilibrium position. The decomposition of the Lagrangian (16), (22) in the vicinity of stationary solution (25) reads

$$
\begin{aligned}
S[u(t), v(t)]=\int L d t, & \\
L= & m\left\{\frac{\omega^{2}}{4 \gamma(k-1)^{2}}+\frac{\beta}{2 \gamma(k-1)} \dot{u}^{2}+\dot{u} \dot{v}+\frac{1}{2 k} \dot{v}^{2}\right. \\
& \left.-\omega^{2}\left(\frac{3}{2(k-1)} u^{2}+u v+\frac{1}{2}(k-1) v^{2}\right)+\ldots\right\} .
\end{aligned}
$$

The dynamical variables are fluctuations in the vicinity of nonzero equilibrium position (25),

$$
u=x \mp \frac{1}{\sqrt{\gamma(k-1)}}, \quad v=y \pm \frac{1}{(k-1) \sqrt{\gamma(k-1)}} .
$$

The dots denote the cubic and higher terms in $u, v$. The generalized momenta $p_{u}, p_{v}$ are defined as follows:

$$
p_{u}=m\left(\frac{\beta}{\gamma(k-1)} \dot{u}+\dot{v}\right), \quad p_{v}=m(\dot{u}+k \dot{v}) .
$$

The generalized momenta $\dot{u}, \dot{v}$ are expressed as follows: 


$$
\begin{aligned}
& \dot{u}=\frac{1}{m} \frac{\gamma(k-1)\left(p_{v}-k p_{u}\right)}{\gamma(k-1)-\beta k} \\
& \dot{v}=\frac{1}{m} \frac{\gamma(k-1) p_{u}-\beta p_{v}}{\gamma(k-1)-\beta k}
\end{aligned}
$$

The Hamiltonian of the model reads

$$
\begin{aligned}
H= & -\frac{m \omega^{2}}{4 \gamma(k-1)^{2}} \\
& +\frac{1}{m} \frac{\gamma(k-1)}{\gamma(k-1)-\beta k}\left(\frac{1}{2} k p_{u}^{2}-p_{u} p_{v}+\frac{1}{2} \frac{\beta}{\gamma(k-1)} p_{v}^{2}\right) \\
& +m \omega^{2}\left(\frac{3}{2(k-1)} u^{2}+u v+\frac{1}{2}(k-1) v^{2}\right) .
\end{aligned}
$$

The Hamiltonian is bounded from below if the conditions (29) are met. This means that the theory of small fluctuations is stable at the classical and quantum level.

The canonical coordinates $v, p_{u}, p_{v}$ are expressed in terms the derivatives of the fluctuation $u$ and its derivatives up to the third order,

$$
\begin{aligned}
& v=\frac{1}{\omega^{2}}\left(1-\frac{\beta k}{\gamma(k-1)}\right) \ddot{u}-\frac{2 k+1}{k-1} u, \\
& p_{u}=\frac{1}{\omega^{2}}\left(1-\frac{\beta k}{\gamma(k-1)}\right) \dddot{u}+\left(\frac{\beta}{\gamma(k-1)}-\frac{2 k+1}{k-1}\right) \dot{u}, \\
& p_{v}=\frac{k}{\omega^{2}}\left(1-\frac{\beta k}{\gamma(k-1)}\right) \dddot{u}-\frac{2 k^{2}+1}{k-1} \dot{u} .
\end{aligned}
$$

The inverse transformation reads [expression for $\dot{u}$ see in (50)]

$$
\begin{aligned}
\ddot{u}= & \omega^{2} \frac{\gamma(k-1) v+\gamma(2 k+1) u}{\gamma(k-1)-\beta k}, \\
\dddot{u}= & \left(\frac{\gamma(k-1)}{\gamma(k-1)-\beta k}\right)^{2} \\
& \times\left(\left(\frac{2 k+1}{k-1}-\frac{\beta}{\gamma(k-1)}\right) p_{v}-\frac{2 k^{2}+1}{k-1} p_{u}\right) .
\end{aligned}
$$

In terms of derivatives of $u$, the Hamiltonian (51) takes the form of integral of motion (27). The Poisson bracket reads

$$
\begin{aligned}
& \{u, \ddot{u}\}=\{\dot{u}, \dddot{u}\}=0, \quad\{u, \dot{u}\}=-\frac{1}{m} \frac{\gamma k(k-1)}{\gamma(k-1)-\beta k}, \\
& \{\ddot{u}, \dddot{u}\}=-\frac{\omega^{4}}{m}\left(\frac{\gamma(k-1)}{\gamma(k-1)-\beta k}\right)^{3}\left(\frac{\beta}{\gamma(k-1)}+\frac{3}{k-1}\right), \\
& \{u, \dddot{u}\}=\{\ddot{u}, \dot{u}\}=\frac{\omega^{2}}{m}\left(\frac{\gamma(k-1)}{\gamma(k-1)-\beta k}\right)^{2} \frac{2 k^{2}+1}{k-1} .
\end{aligned}
$$

The coordinate $u$ and velocity $\dot{u}$ are inevitably have nonzero Poisson bracket for stable interactions. This automatically means that such a Hamiltonian does not follow from the Ostrogradki procedure. This is not surprising because the Ostrogradski Hamiltonian of a nonsingular higher derivative theory is not bounded from below, while the function (51) is bounded.

The example of the higher derivative PU theory (23) with the multiple frequency tells us that the stable vertices are possible, though they are not necessarily Lagrangian. One more conclusion is that the higher derivative equations with non-Lagrangian interactions can admit Hamiltonian formulation, though it is inequivalent to any Ostrogradski formalism. If the quantization of the theory of fluctuations (31) is constructed by the means Hamiltonian formulation (49), (51), the classical stability will persist at quantum level. Being equivalent to the system of two oscillators, this model has the usual equidistant spectrum of energy, and it admits the well-defined vacuum state. The nonharmonic terms can be accounted for by perturbation theory. As the wave functions of stationary states of harmonic oscillator exponentially decreasing at infinity, the perturbation theory is well defined in each order. In principle, this is sufficient for perturbative construction of the stable quantum theory of the nonlinear model (23).

\section{A HIGHER DERIVATIVE FIELD-THEORY WITH THE RESONANCE}

The Podolsky electrodynamics without a Maxwell term provides the simplest example of gauge field theory with resonance. The action reads ${ }^{4}$

$$
S[A(x)]=-\frac{1}{4} \int \partial_{\nu} F^{\nu \mu} \partial^{\rho} F_{\rho \mu} d^{d} x
$$

where $F_{\mu \nu}=\partial_{\mu} A_{\nu}-\partial_{\nu} A_{\mu}$ is the field strength. In the general Podolsky model, the action also includes the Maxwell term, so one of the photons is massless, while another one is massive. The mass spectrum of the model (55) is degenerate: both the subrepresentations are massless. To our knowledge, this theory has not been studied in the literature yet. Maybe it does not attract the interest because the representation with degenerate mass spectrum is nonunitary. We view (55) as a toy model that exemplifies the stability issue in a higher derivative field theory with the resonance at free level, leaving aside the interpretation of the nonunitary representation.

Similar to the PU model (6), the theory (55) admits an equivalent formulation without higher derivatives. The analog of the lower derivative action (1) reads

$$
\mathcal{L}=-\frac{1}{4} k G_{\mu \nu} G^{\mu \nu}-\frac{1}{2} G_{\mu \nu} F^{\mu \nu}+\frac{1}{2} m^{2} B_{\mu} B^{\nu}
$$

\footnotetext{
${ }^{4}$ We use mostly negative convention for the Minkowski metrics.
} 
where $G_{\mu \nu}=\partial_{\mu} B_{\nu}-\partial_{\nu} B_{\mu}$, and $m$ is the constant with mass dimension. We introduce $m$ for reasons of convenience. The dynamical variables are the vector fields $A_{\mu}(x), B_{\mu}(x)$. The Lagrange equations read

$$
\partial^{\mu}\left(F_{\mu \nu}+k G_{\mu \nu}\right)+m^{2} B_{\mu}=0, \quad \partial_{\nu} G^{\mu \nu}=0 .
$$

The first equations defines the vector field $B_{\mu}$ in terms of derivatives of $A, B_{\mu} \approx-m^{-2} \partial^{\nu} F_{\mu \nu}$. Then, the second equation means that $A$ should obey the "double massless" Podolsky equation $\square \partial^{\nu} F_{\nu \mu}=0$. Obviously, the mass $m$ is an accessory parameter that does not contribute to the equations, much like the parameter $k$ in the first order equivalent (1) of the PU action (6).

Much like the mechanical analogue (8) of the previous section, the first-order theory (56) admits a two-parameter series of conserved tensors. So, the higher derivative equivalent (55) should also admit the series of conserved quantities,

$$
\begin{aligned}
\Theta^{\mu \nu}= & -G^{\mu}{ }_{\rho} F^{\nu \rho}-G_{\rho}^{\nu} F^{\mu \rho}+\frac{1}{2} \eta^{\mu \nu} G_{\rho \sigma} F^{\rho \sigma} \\
& -k\left(G_{\rho}^{\mu} G^{\nu \rho}-\frac{1}{4} \eta^{\mu \nu} G_{\rho \sigma} G^{\rho \sigma}\right) \\
& +m^{2}\left(B^{\mu} B^{\nu}-\frac{1}{2} \eta^{\mu \nu} B_{\rho} B^{\rho}\right) .
\end{aligned}
$$

The parameter of the series is the real number $k$. The canonical energy-momentum tensor of the higher derivative theory (55) is included in the series (58) for $k=0$. The general representative of the series (58) is associated with canonical energy-momentum tensor of the theory (56). The energy density is given by the 00-component of the conserved tensor. The energy density is unbounded because the field $A_{\mu}(x)$ is involved into (58) in a linear way. Because of this observation, the theory (56) is unstable at the free level. To stabilize the dynamics at the interacting level, the terms with quadratic dependence on the vector field $A_{\mu}(x)$ are needed. To make it in an explicitly covariant way we include the complex scalar field. From this perspective, we slightly deviate from the pattern of the mechanical model considered in the previous section, where no extra degree of freedom is needed for the stabilization at the interacting level.

The theory (56) of the vector fields $A_{\mu}(x)$ and $B_{\mu}(x)$ admits the following interactions with the complex scalar field $\varphi(x)$ :

$$
\begin{aligned}
\mathcal{L}= & \frac{1}{2}\left(-G_{\mu \nu} F^{\mu \nu}+m^{2} B_{\mu} B^{\nu}-\frac{1}{2} k G_{\mu \nu} G^{\mu \nu}\right) \\
& +D_{\mu} \varphi^{*} D^{\mu} \varphi+m^{2}\left(\alpha|\varphi|^{2}-\frac{1}{2} \beta|\varphi|^{4}\right)-\frac{1}{4} \gamma|\varphi|^{2} F_{\mu \nu} F^{\mu \nu}, \\
D_{\mu} \varphi= & \left(\partial_{\mu}-i e A_{\mu}\right) \varphi, \quad D_{\mu} \varphi^{*}=\left(\partial_{\mu}+i e A_{\mu}\right) \varphi^{*}
\end{aligned}
$$

where $\alpha, \beta, \gamma$ are coupling constants, and $e$ is electric change. The interaction is consistent ${ }^{5}$ because the Lagrangian is invariant with respect to the usual $U(1)$ gauge transformations. The interacting theory (59) corresponds to the pattern of interaction for the mechanical model (16) with a nonzero function $U$, which depends on the original vector field and the complex scalar $\varphi(x)$. The inclusion of the scalar field is essential. The Born-Infieldtype interactions, which are expressed in terms of the higher degrees of strength tensor $F_{\mu \nu}$, are irrelevant to stability because the energy of the model is unbounded in the linear approximation. The model (59) describes the nonminimal couplings of the vector field and complex scalar field, being tachyon at free level. The $|\varphi|^{4}$ stabilizes the dynamics of scalar field, while the term $|\varphi|^{2} F_{\mu \nu} F^{\mu \nu}$ dynamically generates the mass of the vector field.

The theory (59) corresponds to the model of the single higher derivative vector field $A_{\mu}(x)$ and scalar field $\varphi(x)$. The field equations read

$$
\begin{aligned}
\frac{\delta S}{\delta \varphi^{*}} & =\left(D^{\mu} D_{\mu}-\alpha+\beta|\varphi|^{2}+\frac{1}{4} \gamma F_{\rho \sigma} F^{\rho \sigma}\right) \varphi=0, \\
\frac{\delta S}{\delta \varphi} & =\left(D^{\mu} D_{\mu}-\alpha+\beta|\varphi|^{2}+\frac{1}{4} \gamma F_{\rho \sigma} F^{\rho \sigma}\right) \varphi^{*}=0, \\
\frac{\delta S}{\delta A^{\mu}} & =\partial^{\mu}\left(G_{\mu \nu}+\gamma|\varphi|^{2} F_{\mu \nu}\right)-j_{\nu}(\varphi, A)=0, \\
\frac{\delta S}{\delta B^{\mu}} & =\partial^{\mu}\left(F_{\mu \nu}+k G_{\mu \nu}\right)+m^{2} B_{\nu}=0,
\end{aligned}
$$

where $j_{\mu}(\varphi, A)$ denotes the scalar field charge,

$$
j_{\mu}(\varphi, A)=-i e\left(\varphi^{*} D_{\mu} \varphi-\varphi D_{\mu} \varphi^{*}\right) .
$$

From these equations, the vector $B_{\mu}(x)$ can be expressed on shell, $B_{\mu} \approx m^{-2}\left[-\partial^{\nu}\left(F_{\nu \mu}+k \gamma|\varphi|^{2} F_{\mu \nu}\right)-k j_{\mu}(\varphi, A)\right]$. Substituting the result into the remaining equations and accounting for $\partial_{\mu} j^{\mu} \approx 0$, we obtain

$$
\begin{aligned}
&-\square \partial^{\mu} F_{\mu \nu}+\left(k \square+m^{2}\right)\left(\partial^{\mu}\left(\gamma|\varphi|^{2} F_{\mu \nu}\right)-j_{\nu}(\varphi, A)\right)=0, \\
&\left(D^{\mu} D_{\mu}-\alpha+\beta|\varphi|^{2}+\frac{1}{4} \gamma F_{\rho \sigma} F^{\rho \sigma}\right) \varphi=0, \\
&\left(D^{\mu} D_{\mu}-\alpha+\beta|\varphi|^{2}+\frac{1}{4} \gamma F_{\rho \sigma} F^{\rho \sigma}\right) \varphi^{*}=0 .
\end{aligned}
$$

These equations are non-Lagrangian if $k \neq 0$. The conserved tensor of the model (59) is the canonical energymomentum tensor, i.e.,

\footnotetext{
${ }^{5}$ The concept of consistency of interaction in the variational formalism is reviewed in [31]. For consistency of interactions for not necessarily Lagrangian equations, see [32].
} 


$$
\begin{aligned}
\Theta^{\mu \nu}= & -G_{\rho}^{\mu} F^{\nu \rho}-G_{\rho}^{\nu} F^{\mu \rho}+\frac{1}{2} \eta^{\mu \nu} G_{\rho \sigma} F^{\rho \sigma}-k\left(G_{\rho}^{\mu} G^{\nu \rho}\right. \\
& \left.-\frac{1}{4} \eta^{\mu \nu} G_{\rho \sigma} G^{\rho \sigma}\right)+m^{2}\left(B^{\mu} B^{\nu}-\frac{1}{2} \eta^{\mu \nu} B_{\rho} B^{\rho}\right) \\
& -\gamma|\varphi|^{2}\left(F_{\rho}^{\mu} F^{\nu \rho}-\frac{1}{4} \eta^{\mu \nu} F_{\rho \sigma} F^{\rho \sigma}\right) \\
& +D^{\mu} \varphi^{*} D^{\nu} \varphi+D^{\nu} \varphi^{*} D^{\mu} \varphi \\
& -\eta^{\mu \nu} D^{\rho} \varphi^{*} D_{\rho} \varphi-\alpha m^{2}|\varphi|^{2}+\frac{1}{2} \beta m^{2}|\varphi|^{4}
\end{aligned}
$$

The conserved tensor of the model (62) is deduced from the expression above by expressing the auxiliary field $B_{\mu}(x)$ in terms of the derivatives of $A_{\mu}(x)$ on shell.

Consider the issue of stability of the theory (62). The model has a nonzero stationary solution,

$$
A_{\mu}(x)=0, \quad \varphi(x)=e^{i \theta} \sqrt{\frac{\alpha}{\beta}},
$$

where $\theta$ is the angle of the vacuum, and $\alpha, \beta$ are interaction parameters. In the vicinity of this solution, the conserved tensor (63) reads

$$
\begin{aligned}
\Theta^{\mu \nu}= & -G^{\mu}{ }_{\rho} F^{\nu \rho}-G_{\rho}^{\nu} F^{\mu \rho}+\frac{1}{2} \eta^{\mu \nu} G_{\rho \sigma} F^{\rho \sigma}-k\left(G_{\rho}^{\mu} G^{\nu \rho}\right. \\
& \left.-\frac{1}{4} \eta^{\mu \nu} G_{\rho \sigma} G^{\rho \sigma}\right)+m^{2}\left(B^{\mu} B^{\nu}-\frac{1}{2} \eta^{\mu \nu} B_{\rho} B^{\rho}\right)-\frac{\gamma \alpha}{\beta} \\
& \times\left(F^{\mu}{ }_{\rho} F^{\nu \rho}-\frac{1}{4} \eta^{\mu \nu} F_{\rho \sigma} F^{\rho \sigma}\right)+\partial^{\mu} \phi^{*} \partial^{\nu} \phi+\partial^{\nu} \phi^{*} \partial^{\mu} \phi \\
& -\eta^{\mu \nu} \partial^{\phi} \phi^{*} \partial_{\rho} \phi+\alpha m^{2}\left(e^{i \theta} \phi+e^{-i \theta} \phi^{*}\right)^{2}
\end{aligned}
$$

where $\phi(x)=\varphi(x)-e^{i \theta} \sqrt{\alpha / \beta}$ is the scalar field fluctuation, and $B_{\mu}=\partial^{\nu} F_{\mu \nu}$. The 00-component of the conserved tensor $(65)$ reads

$$
\begin{aligned}
\Theta^{00}= & G^{i} F^{i}+G^{i j} F^{i j}+\frac{\gamma \alpha}{2 \beta}\left(F^{i} F^{i}+F^{i j} F^{i j}\right)+\frac{k}{2}\left(G^{i} G^{i}\right. \\
& \left.+G^{i j} G^{i j}\right)+\frac{1}{2} m^{2}\left(B^{0} B^{0}+B^{i} B^{i}\right)+\partial^{0} \phi^{*} \partial^{0} \phi \\
& +\partial^{i} \phi^{*} \partial^{i} \phi+\alpha m^{2}\left(e^{i \theta} \phi+e^{-i \theta} \phi^{*}\right)^{2}
\end{aligned}
$$

where $F^{i}=F^{0 i}, G^{i}=G^{0 i}$. The summation over the repeated index $i, j=1, \ldots, d-1$ is implied. The quadratic form $\Theta^{00}$ is positive definite if

$$
\frac{k \gamma \alpha}{\beta}-1>0 .
$$

In this range of the coupling parameters $\alpha, \beta, \gamma$, the interacting theory of small fluctuations in the vicinity of stationary solution (64) is stable. We note that for the stable interactions the parameter $k$ should be strictly positive, so the higher derivative theory (62) is inevitably nonLagrangian at interacting level.

Let us discuss the dynamics of the stable theory (62), (67). The linearization of equations of motion (62) in the vicinity of stationary solution (64) takes the following form:

$$
\begin{aligned}
\left(\left(\frac{k \gamma \alpha}{\beta}-1\right) \square+m^{2} \frac{\alpha}{\beta}\right) \partial^{\nu} F_{\nu \mu} & =0, \\
\square \phi+\alpha m^{2}\left(\phi+e^{2 i \theta} \phi^{*}\right) & =0 .
\end{aligned}
$$

In the sector of vector fields, we have the usual Podolsky theory without a resonance. The spectrum of the mass of the vector field theory includes the massless state, and the massive state with the mass $m \sqrt{\alpha /(k \alpha \gamma-\beta)}$. This theory is stable and unitary at free level; see in [22]. The complex scalar field is decomposed into two real components $\phi^{+}=e^{-i \theta} \phi+e^{i \theta} \phi^{*}$ and $\phi^{-}=$ $i\left(e^{-i \theta} \phi-e^{i \theta} \phi^{*}\right)$. The scalars enjoy the Klein-Gordon and d'Alembert equations, respectively,

$$
\begin{aligned}
\left(\square+2 \alpha m^{2}\right) \phi^{+} & =0, \\
\square \phi^{-} & =0 .
\end{aligned}
$$

The mass of the first field is $\sqrt{2 \alpha} \mathrm{m}$, while the second one is massless. Summarizing all the above, we conclude that the solutions of the Eq. (68) transform under the unitary representation of the Poincaré group. The set of subrepresentations includes the massive vector and scalar, and massless vector and scalar. The role of the scalar field is seen from comparison of Eqs. (62) and (68). The nonzero value of the scalar field $\varphi$ generates the mass term for the vector field. The similar mechanism is served by the Higgs field in the standard model. With this regard, the above method of construction of stable interactions in the higher derivative field theories with resonance can be viewed as the Higgs-like mechanism.

Let us comment on the "Higgs mechanism" of stabilizing the higher derivative dynamics from slightly different point of view, being unrelated to the existence of the resonant solutions. It is a common wisdom that the PU oscillator with nondegenerate frequency spectrum is equivalent to the system of free harmonic oscillators. The canonical Ostrogradski's energy of the PU action includes the energies of these oscillators with the opposite signs. This corresponds to the canonical energy of the first order action, being a combination of harmonic oscillators with the alternating signs. A corresponding Lagrangian admits the interaction vertices such that the corresponding energy has a local minimum, so the dynamics is stable in the vicinity of this shifted equilibrium point. The Lagrangian reads 


$$
\begin{aligned}
L= & m\left(\frac{1}{2}\left(\dot{x}^{2}-\omega_{x}^{2} x^{2}\right)-\frac{1}{2}\left(\dot{y}^{2}-\omega_{y}^{2} y^{2}\right)+\frac{1}{2}\left(\dot{z}^{2}+\omega_{z}^{2} z^{2}\right)\right. \\
& \left.-\frac{1}{4} \alpha \omega_{z}^{2} z^{4}+\frac{1}{2} \beta z^{2}\left(\dot{y}^{2}-\omega_{y}^{2} y^{2}\right)\right)
\end{aligned}
$$

where $\omega_{x}, \omega_{y}, \omega_{z}$ are the frequencies, $\omega_{x} \neq \omega_{y}$, and $\alpha$, $\beta>0$ are the coupling constants. The variables $x, y$ can be thought of as the degrees of freedom of the original PU oscillator, while $z$ can be viewed as the "Higgs mode." Upon inclusion of the interaction, the equilibrium position shifts to the point $x, y=0, z=\sqrt{1 / \alpha}$. If $\beta / \alpha>1$, the energy is positive in the vicinity of the equilibrium position. This indicates the stability in the vicinity of the equilibrium. Once the frequency spectrum is nondegenerate, the PU equations admit globally stable interactions [22] unlike the degenerate case, while the Higgs-like mechanism results in the local stability in both cases.

\section{HIGHER DERIVATIVE YANG-MILLS THEORY}

In the previous section we considered a mechanism for including the stable interaction in the Abelian higher derivative gauge theory. The key tool for doing that is the series of equivalent actions (56) without higher derivatives involving the parameter $k$ which does not contribute to the equations at free level. The action (56) admits inclusion of $k$-dependent stable interaction (59) such that the auxiliary fields $B$ can be still excluded on shell. This leads to the field equations with stable interactions, though the vertices are not Lagrangian in the higher derivative picture. This mechanism admits, to some extent, a nonAbelian extension. At the level of the first order action (59), the non-Abelian generalization is obvious,

$$
\begin{aligned}
\mathcal{L}= & \frac{1}{2}\left(-G_{\mu \nu}^{a} F^{a \mu \nu}+m^{2} B_{\mu}^{a} B^{a \mu}-\frac{1}{2} k G_{\mu \nu}^{a} G^{a \mu \nu}\right) \\
& +\frac{1}{2}\left(D_{\mu} \varphi^{a} D^{\mu} \varphi^{a}+m^{2}\left(\alpha \varphi^{a} \varphi^{a}-\frac{1}{2} \beta\left|\varphi^{a} \varphi^{a}\right|^{2}\right)\right) \\
& -\frac{1}{4} \gamma \varphi^{a} \varphi^{a} F_{\mu \nu}^{b} F^{b \mu \nu} .
\end{aligned}
$$

Here, all the scalars and vectors take values in Lie algebra of a certain semisimple Lie group of dimension $r$, $a=1, \ldots, r$. The tensor $F$ denotes the Yang-Mills strength tensor of the field $A$. The tensors $G$ is the field strength of the vector field $B, G_{\mu \nu}=D_{\mu} B_{\nu}-D_{\nu} B_{\mu}$. The Yang-Mills covariant derivative is $D=\partial+A$. The Lagrangian is invariant with respect to the Yang-Mills gauge symmetry transformation. The vector multiplet $A$ transforms as connection. The vector $B$ and scalar $\varphi$ transform as tensors. The canonical energy is bounded for the model (71) admits a local minimum. The decomposition of Lagrangian in the vicinity of the energy minimum has the same structure as in the Abelian case (62). This theory has same stability conditions (67). The only subtlety is that the auxiliary field $B$ cannot be explicitly expressed from the equations of motion unlike the Abelian case. This can be done only by perturbation theory with respect to the Yang-Mills coupling constant. Once the auxiliary field $B$ is expressed from the equations of motion, the non-Lagrangian non-Abelian higher derivative gauge theory, which is stable and unitary.

\section{CONCLUSION}

In this article, we consider the issue of stability in the class of the higher derivative theories of derived type with a resonance. The wave operator of the theory is the square of another lower-order operator. We see that this class of models admits a two-parameter series of conserved quantities. One of the entries of the series is the canonical energy, and another one is a different integral of motion. All the conserved quantities are unbounded. This structure of conserved quantities is consistent with the instability of free model. To stabilize the dynamics of theory at the nonlinear level, the class of interactions is considered such that preserves the selected conserved quantity of the free model. The conserved quantity of the nonlinear theory is bounded from below in the vicinity of the equilibrium due to the interaction. Therefore, the fluctuations are stable in vicinity of the equilibrium. The stable interactions are nonLagrangian in the higher derivative equations, but the dynamics admit the Hamiltonian formulation. The Hamiltonian is defined by the conserved quantity of the interacting theory. Being bounded from below, the Hamiltonian is not canonically equivalent to any (deformation of the) Ostrogradski one.

The general scheme is illustrated by the PU oscillator of fourth order with coinciding frequencies and by Podolsky electrodynamics with zero mass. Both models are unstable at the free level, but they can be stabilized by an appropriate interaction. Explicit examples are provided of the stable interactions. The Hamiltonian form of dynamics and size of the stability island are found in the case of the PU oscillator model. The Hamiltonian and Poisson bracket are explicitly derived for the model with interaction, and the Hamiltonian is locally bounded. The PU oscillator dynamics with stabile interactions are also described in the original set of variables. In the Podolsky theory, the Higgs field, being the charged scalar, is introduced to explicitly preserve gauge invariance. The interacting model is a theory of higher derivative vector field nonminimally coupled to the charged scalar. The Higgs field has nonzero value at the minimum of energy. The theory of small fluctuations in the vicinity of the energy minimum has a nondegenerate spectrum of mass. The model with nondegenerate mass spectrum is shown to be stable and unitary.

The proposed procedure of inclusion of stable interactions seems admitting further applications. It is consistent 
with the non-Abelian gauge symmetry, and it can be used in the theories of PU type without resonance. Among the possible applications, we can mention various higher derivative models of interest, including gravity. The common feature is that these theories are unstable at free and interacting level (except the class of $f(R)$-gravity models). The introduction of appropriate set of Higgs-type fields, can, in principle, stabilize the dynamics of the theory as we have seen in this article.

\section{ACKNOWLEDGMENTS}

We thank A. A. Sharapov for discussions on various issues addressed in this work. The work of D. S. Kaparulin is supported by the Russian Science Foundation Grant No. 18- 72-10123 in association with the Lebedev Physical Institute of RAS. S. L. Lyakhovich is benefited from a government task of the Ministry of Science and Higher Education of the Russian Federation, Project No. 07212020-0033.
[1] A. Pais and G. E. Uhlenbeck, On field theories with nonlocalized action, Phys. Rev. 79, 145 (1950).

[2] V. A. Abakumova, D. S. Kaparulin, and S. L. Lyakhovich, Multi-Hamiltonian formulations and stability of higherderivative extensions of $3 \mathrm{~d}$ Chern-Simons, Eur. Phys. J. C 78, 115 (2018).

[3] B. Podolsky, A generalized electrodynamics. Part I-nonquantum, Phys. Rev. 62, 68 (1942).

[4] H. Lu, Yi Pang, and C. N. Pope, Conformal gravity and extensions of critical gravity, Phys. Rev. D 84, 064001 (2011).

[5] H. Lu, Yi Pang, and C. N. Pope, Black holes in sixdimensional conformal gravity, Phys. Rev. D 87, 104013 (2013).

[6] S. Deser and R. Jackiw, Higher derivative Chern-Simons extensions, Phys. Lett. B 451, 73 (1999).

[7] E. T. Tomboulis, Renormalization and unitarity in higher derivative and nonlocal gravity theories, Mod. Phys. Lett. A 30, 1540005 (2015).

[8] A. V. Smilga, Classical and quantum dynamics of higherderivative systems, Int. J. Mod. Phys. A 32, 1730025 (2017).

[9] G. W. Gibbons, C. N. Pope, and S. Solodukhin, Higher derivative scalar quantum field theory in curved spacetime, Phys. Rev. D 100, 105008 (2019).

[10] M. Cvetic, G. W. Gibbons, C. N. Pope, and B. F. Whiting, Positive energy functional for massless scalars in rotating STU black hole backgrounds, arXiv:1912 .08988 .

[11] C. M. Bender and P. D. Mannheim, No-Ghost Theorem for the Fourth-Order Derivative Pais-Uhlenbeck Oscillator Model, Phys. Rev. Lett. 100, 110402 (2008).

[12] C. M. Bender, Giving up the ghost, J. Phys. A 41, 304018 (2008).

[13] A. Mostafazadeh, A Hamiltonian formulation of the PaisUhlenbeck oscillator that yields a stable and unitary quantum system, Phys. Lett. A 375, 93 (2010).

[14] K. Bolonek and P. Kosinski, Hamiltonian structures for Pais-Uhlenbeck oscillator, Acta Phys. Pol. B 36, 2115 (2005), https://www.actaphys.uj.edu.pl/fulltext?series=Reg\& vol $=36 \&$ page $=2115$.
[15] E. V. Damaskinsky and M. A. Sokolov, Remarks on quantization of Pais-Uhlenbeck oscillators, J. Phys. A 39, 10499 (2006).

[16] A. V. Smilga, Benign vs malicious ghosts in higherderivative theories, Nucl. Phys. B706, 598 (2005).

[17] A. V. Smilga, Comments on the dynamics of the PaisUhlenbeck oscillator, SIGMA 5, 017 (2009).

[18] M. Pavsic, Pais-Uhlenbeck oscillator and negative energies, Int. J. Geom. Methhods Mod. Phys. 13, 1630015 (2016).

[19] M. Avendaco-Camacho, J. A. Vallejo, and Y. Vorobiev, A perturbation theory approach to the stability of the PaisUhlenbeck oscillator, J. Math. Phys. (N.Y.) 58, 093501 (2017).

[20] N. Boulanger, F. Buisseret, F. Dierick, and O. White, Higher-derivative harmonic oscillators: Stability of classical dynamics and adiabatic invariants, Eur. Phys. J. C 79, 60 (2019).

[21] A. Salvio, Metastability in quadratic gravity, Phys. Rev. D 99, 103507 (2019).

[22] D. S. Kaparulin, S. L. Lyakhovich, and A. A. Sharapov, Classical and quantum stability of higher-derivative dynamics, Eur. Phys. J. C 74, 3072 (2014).

[23] D. S. Kaparulin, I. Yu. Karataeva, and S. L. Lyakhovich, Higher derivative extensions of $3 \mathrm{~d}$ Chern-Simons models: Conservation laws and stability, Eur. Phys. J. C 75, 552 (2015).

[24] D. S. Kaparulin and S. L. Lyakhovich, Energy and stability of the Pais-Uhlenbeck osciallator, Proceedings of the XXXIII Workshop on Geometric Methods in Physics (Springer, New York, 2015), pp. 127-134.

[25] D. S. Kaparulin, S. L. Lyakhovich, and A. A. Sharapov, Stable interactions via proper deformations, J. Phys. A 49, 155204 (2016).

[26] D. S. Kaparulin, I. Y. Karataeva, and S. L. Lyakhovich, Third order extensions of 3d Chern-Simons interacting to gravity: Hamiltonian formalism and stability, Nucl. Phys. B934, 634 (2018).

[27] V. A. Abakumova, D. S. Kaparulin, and S. L. Lyakhovich, Stable interactions in higher derivative field theories of derived type, Phys. Rev. D 99, 045020 (2019).

[28] D. S. Kaparulin and S. L. Lyakhovich, On stability of nonlinear oscillator with higher derivatives, Russ. Phys. J. 57, 1561 (2015). 
[29] M. V. Ostrogradski, Memoires sur les equations differentielles relatives au probleme des isoperimetretres, Mem. Acad. St. Petersburg 6, 385 (1850).

[30] D. M. Gitman, S. L. Lyakhovich, and I. V. Tyutin, Hamilton formulation of a theory with high derivatives, Sov. Phys. J. 26, 730 (1983).
[31] M. Henneaux, Consistent interactions between gauge fields: The cohomological approach, Contemp. Math. 219, 93 (1998).

[32] D. S. Kaparulin, S. L. Lyakhovich, and A. A. Sharapov, Consistent interactions and involution, J. High Energy Phys. 01 (2013) 097. 